\title{
HARMONIC MULTIRESOLUTION ESTIMATORS FOR DENOISING AND REGULARIZATION OF SPECT-PET DATA
}

\author{
Elsa D. Angelini, Jérôme Kalifa, Andrew F. Laine \\ Department of Biomedical Engineering, Columbia University, New York, NY, USA
}

\begin{abstract}
This paper presents a study on the development of new multiresolution directional analysis tools for texture denoising of medical images. Multiresolution texture analysis is performed with wavelet packets and brushlet expansions to exploit spatio-temporal coherence and identify persistent anatomical structures while removing uncorrelated noise components. Denoising is performed via thresholding estimators in the transform domain. Denoising performance is evaluated quantitatively on phantom volumes and qualitatively on clinical data sets with SPECT-PET data. We show in this study that these multiresolution directional analysis tools are well adapted to the intrinsic nature of textured data and outperform traditional denoising methods. In the case of spatiotemporal data, we also show that by incorporating the time dimension directly in the analysis, we can bring into play temporal coherence between successive frames to improve denoising performance and enhance moving boundaries and structures.
\end{abstract}

\section{INTRODUCTION}

This paper presents a new study on multiscale analysis for texture denoising tailored to medical imaging modalities corrupted with textured noise. To illustrate the performance of our algorithm we focus on PET and SPECT nuclear images where noise is introduced in the process of an ill-defined reconstruction process. In this case, noise components result in the addition of textural patterns that degrade the quality of the diagnostic image and make visual inspection very difficult. These textures also prevent simple analysis tools and quantitative measures from performing accurately.

In image processing, textures are commonly characterized by their orientation and their frequency. Such patterns are well depicted by the eye but quite difficult to characterize quantitatively. We propose in this study a new type of denoising based on multiresolution linear thresholding estimators targeted for textured noisy images focusing on two families of multiscale analysis functions: (1) wavelet packets (WP) and (2) brushlet functions. These two families perform a compact tiling of the Fourier domain and separately analyze sub-Fourier domains associated with textural patterns of distinct orientations and frequencies.

\subsection{SPECT-PET tomography}

Tomographic image modalities such as PET and SPECT rely on an instable inverse problem of spatial signal reconstruction from sampled line projections. Because the Radon transform is a smoothing process, back projection in the presence of additive noise is an ill-posed inverse problem that requires a regularization of the reconstructed noise component, which can become very large. Standard regularization methods include filtered back-projection (FBP) with non-linear filtering corrections, expectationmaximization (EM) and maximum a posteriori (MAP) estimators [1-4]. To address the limitations of these existing methods in terms of poor performance, instability and computational complexity, recent work has focused on a new family of regularizing methods based on wavelet analysis [5, 6]. In our approach, regularization is converted to a denoising problem performed via thresholding of WP coefficients. In an earlier study, Blair et al. applied wavelet thresholding to Poisson distributed sinogram data as a regularization tool prior to an EM reconstruction [7]. We present a new regularization method that aims to provide better reconstructed tomographic images with a sound theoretical framework for which the estimation error is understood and characterized, while using fast and flexible implementations.

\section{METHODOLOGY}

Artifacts that corrupt SPECT-PET data lead to images that can be characterized as texture images. Development of analysis tools for texture analysis can be classified into two general methods: statistical analysis and multiresolution analysis. Most popular statistical models were derived from Markov random field theory [8], characterizing texture from statistical interaction with local neighborhoods in the spatial domain. Such statistical models perform poorly on low-resolution images such as with SPECT-PET data. More recent approaches were developed using oriented multiresolution analysis [9]. In this framework, WP, Gabor functions, local cosine basis and steerable pyramid filters have been widely used for texture characterization. These analysis tools suffer from two major limitations: (1) Frequency resolution is 
Presented at the IEEE International Symposium on Biomedical Imaging, Washington D.C., USA, 2002.

constrained to octave bandwidths for efficient implementation and, (2) analysis is not orthogonal due to overlap of the frequency subbands. The difference in sampling rates along the different dimensions suggested that tiling of the space-frequency domain should not be limited to dyadic scales or octave frequency bandwidths. In denoising and enhancement applications, orthogonality of the transform enables decorrelation of noise and signal components in different sub-sets of coefficients. Nonorthogonal analysis functions lead to correlation between texture features and are not suitable for noise removal via coefficient thresholding. For this reason, we tested in this study both WP and orthogonal brushlet basis functions [10]. Denoising is performed via non-linear thresholding. The quality of a non-linear estimator depends on two factors: (1) The aptitude of the basis to decorrelate noise from signal components in independent sets of coefficients, (2) The thresholding operator and the threshold level. We detail these two points in the context of texture denoising.

\subsection{Multiscale analysis function}

\subsubsection{Wavelet Packet}

In the case of SPECT-PET images, the signal is modeled as a spatially inhomogeneous, piece-wise regular signal that can be compactly represented with wavelet decompositions. Donoho suggested first the use of thresholding estimators in wavelet basis for linear inverse problems including the inversion of the Radon transform [11]. In situations where the noisy component of an observation signal is highly texturized, wavelet packets are more appropriate than wavelet functions as they provide a complete segmentation of the frequency domain.

\subsubsection{Brushlets}

The family of wavelet-packet like functions called brushlets constitutes an orthonormal basis of the real axis with arbitrary size support. Brushlet basis functions decompose a signal along specific spatial directions via analysis of its Fourier domain, making the decomposition invariant to the intensity or contrast range of the data.

Wavelet packets and brushlet basis functions are very similar in their structures. The major difference between the two multiresolution analysis tools is the ability of the brushlet to operate on an arbitrary tiling of the timefrequency plane and the perfect localization of a single frequency in each coefficient. This property makes brushlet analysis well adapted for representing textured noise components. On the other hand, WP are better suited for data representation offering a finer representation of piecewise smooth signals. These observations suggested that both methods could be combined for denoising of low-resoltion medical images with highly textured noise components. We report on innovative testing and optimization results in this direction applied to both phantom and clinical data.

\subsubsection{Multidimensional analysis}

Wavelet packets and brushlet analysis are easily extended to $N$ dimensions using tensor products. In this study we have implemented a brushlet expansion in $3 \mathrm{D}$ and a WP expansion is extended to $3 \mathrm{D}$ as well.

\subsection{Thresholding Operator}

In the case of SPECT-PET data, we used a Poisson distributed noise model applied to the original sinogram for estimating the noise variance. This noise estimation process is quite involved and detailed in [12]. After estimating the characterizing Poisson parameter at each node of the WP expansion using the original sinogram data, we use these values as an estimate of the noise variance for minimax thresholding [13]. It is important to note here that the filtered back noise component is not assumed to be Poisson but the variance estimated with this prior model remains consistent at each node of the WP expansion, because of diagonalization in the transform domain. After performing empirical testing on phantoms and SPECT clinical data, we concluded that minimax hard-thresholding performed better than non-adaptive hard and soft thresholding at removing both SPECT-PET and speckle noise while enhancing anatomical structures.

\section{RESULTS}

\subsection{Denoising on Phantom data}

In order to evaluate the performance of the denoising methods we first performed tests on a phantom data set. The phantom consisted of a smooth 2D shape slowly deforming in the third dimension so that we can consider the phantom data as a $3 \mathrm{D}$ or (2D+Time) set. In order to mimic SPECT-PET noise we extracted from a clinical data set a slice without structure. The extracted noisy slice displayed diagonal structure and was fused with the original 3D phantom to obtain a SPECT-PET like noisy phantom. Denoising was tested with both WP and brushlet analysis functions using two distinct strategies for extracting noise and signal components.

For WP, the basis was selected via a best basis search with SURE cost metrics [14] using our prior noise variance estimation. For brushlets, a tiling into four subintervals was applied to each dimension.

Hard thresholding was applied at the following percentage of coefficients maxima: $50 \%$ for 2D WP spatial denoising, $15 \%$ for brushlet temporal denoising in time and $30 \%$ for brushlet temporal denoising in frequency as described in [15]. Each subset of coefficients was denoised separately. These threshold levels were set empirically after experimentation.

Denoising was evaluated both visually and quantitatively with signal to noise ratio (SNR) measures. 


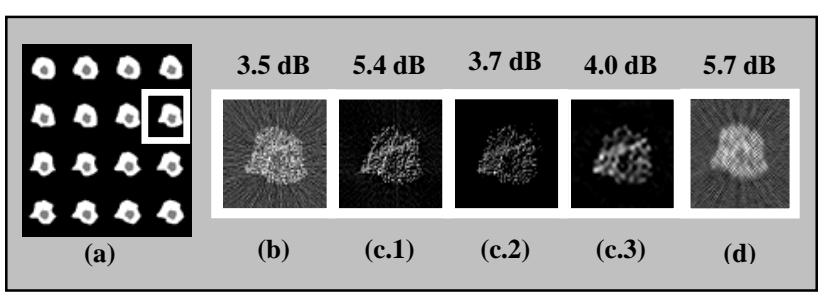

Figure 1: (a) Mathematical phantom with a 3D smooth shape. Slice \#8 is highlighted (b) Slice \#8 extracted from phantom corrupted with SPECT-PET noise. (b.1) Slice (a) denoised in space after $2 D$ brushlet analysis with minimax hard thresholding. (b.2) Slice (b.1) further denoised in time via temporal analysis. (b.3) Slice (b.2) further denoised in time via frequency analysis. (c) Slice (a) denoised with hard thresholding on wavelet packets expansion. SNR values are reported for each case.

Denoising results are displayed in Figure 1 above. With brushlet denoising, we observed that the noisy background was well eliminated but the white texture of the phantom was poorly reconstructed. When using WP, the noisy background was still present, limiting the contrast of the reconstructed image, but the white texture was much better restored. We observed a significant improvement in SNR with each denoising method.

These tests on phantom data showed potentials of both WP and brushlet denoising for removing textured signals. We then performed experiments on clinical data sets using the thresholding operators that performed best on phantom data.

\subsection{Denoising on Clinical data}

The SPECT data set used in this study consisted of a brain sinogram of dimension $(128 \times 128 \times 128)$ reconstructed with a discrete inverse Radon transform. Denoising with WP followed the same procedure as the phantom data, using a pre-computed estimation of the noise variance. Processing with brushlet basis functions required more testing to optimize the quality of the denoising. When testing with minimax hard thresholding, the resulting image was either too smoothed or the brain tissue remained too granular. The reason for this poor performance was that no tiling could be identified that separated correctly noise and signal texture components. Since the frequency content of the noisy background was similar to the structural information when compared to the overall resolution of the data. For this reason we tested a new adaptive thresholding approach initially suggested by Chang et al. [16]. This approach identifies in the noisy image three regions for edges, smooth background and texture (defined as non edges and non smooth) and applies different threshold levels inside the three regions. Because of the overcomplete structure of our brushlet implementation, this methodology could be applied in a straightforward manner and greatly improved the performance of the denoising procedure. Again the threshold levels were empirically set with high values on smooth regions and smaller values on texture and edges regions.

Results are displayed in Figure 2 below, for a set of four slices through the entire volume. We also include in this figure denoising with a standard filtered back projection (FBP) algorithm for comparison purposes. We observed that both WP and brushlet denoising visually outperformed the FBP denoising method but produced quite different images. The WP denoising better preserved the texture of the brain tissue whereas brushlet denoising better eliminated the directional noisy background. It is also interesting to note that brushlet denoising revealed better localized bright spots inside the white matter which can be meaningful for diagnosis.

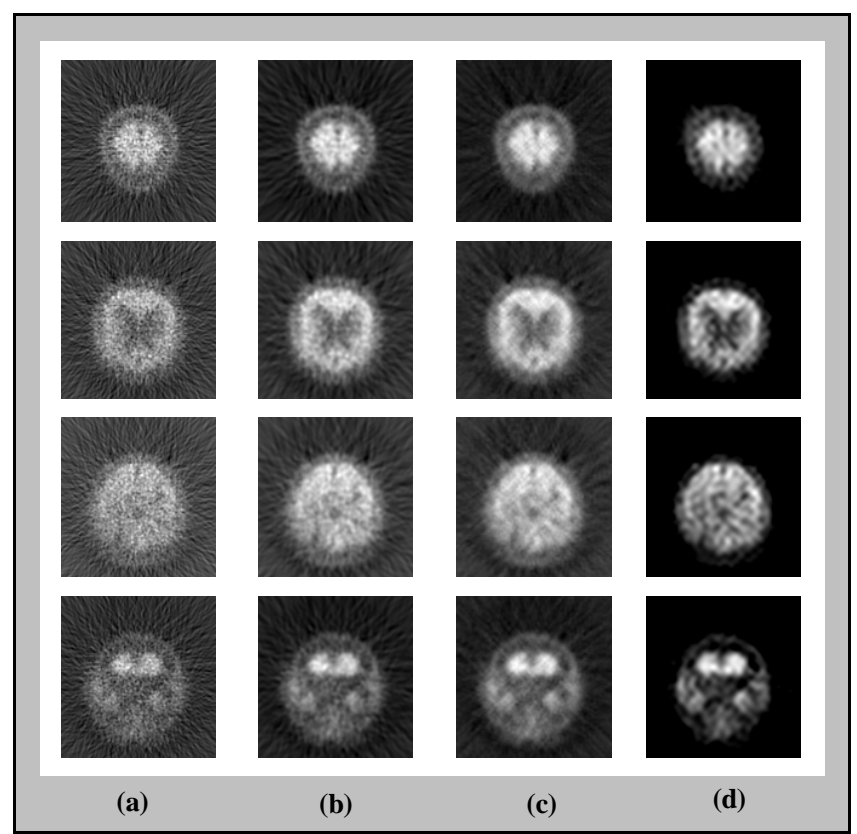

Figure 2. Denoising of SPECT brain data with set of 'best' parameters for FBP, WP and brushlet. Four slices were extracted from the (a) original volume and $(b-d)$ denoised volumes. Denoising was performed with (b) filtered back projection, (c) Wavelet packets and (d) brushlet analysis.

\section{DISCUSSION}

Performance of wavelet packets and brushlet thresholding to decorrelate signal from SPECT-PET noise components in space was demonstrated on mathematical phantoms and clinical SPECT-PET data. We tested analysis and thresholding parameters to identify the set of parameters that 'best' performed at removing noise and enhancing coherent structures. The identification of the set of 'best' parameters was performed both quantitatively on phantom data with SNR measurements and qualitatively on clinical 
Presented at the IEEE International Symposium on Biomedical Imaging, Washington D.C., USA, 2002.

data. We used WP and brushlet basis functions to decompose the signal along oriented textural patterns. Linear estimators using WP expansions resulted in a good reconstruction of piecewise smooth anatomical structures. Linear estimators using brushlet expansion was best suited for eliminating directional texture patterns along selected orientations and resolutions. The results presented on SPECT brain data suggested that wavelet packets and brushlet linear estimators should be combined for optimal results. Further testing of the presented denoising procedures will be carried out for quantitative validations. Validation of denoising quality in SPECT-PET clinical data is a very delicate task since there is no reference to compare to and visual quality assessment can vary greatly among doctors. The methodology of reference to assess such denoising procedures is to perform an ROC study with several users, performing specific diagnosis evaluations on a set of cases and is currently under development [17].

We will also test the denoising method on additional textured medical screening diagnostic modalities such as cardiac three-dimensional ultrasound, cardiac PET and NMR spectroscopy.

\section{CONCLUSION}

We demonstrated the performance of linear estimators to eliminate textured noise while enhancing coherent anatomical structures on PET-SPECT tomographic images. Denoising with multiresolution directional expansions performed better than standard methods of reference, and show great promise in removing textured noise for this low-resolution medical image modality. The denoising tool was derived from a sound theoretical and mathematical framework while using fast and flexible implementations. Prior noise statistics estimations were introduced to optimize parameter settings and tune the processing to a specific denoising or regularization task. We believe that further testing on a wide range of textured medical screening modalities, using a combination of the proposed multiresolution texture-oriented linear estimators, will validate this tool as a powerful and flexible denoising method, exceedingly superior to existing filtering algorithms.

\section{REFERENCES}

[1] G. J. McLachlan and T. Krishnan, The EM Algorithm and Extensions. New York: John and Wiley \& Sons, Inc, 1997.

[2] L. A. Shepp and Y. Vardi, "Maximum likelihood reconstruction for emission tomography," IEEE Transactions on Medical Imaging, pp. 113-122, 1982.

[3] T. H. Farquhar, A. Chatziioannou, G. Chinn, M. Dahlbom, and E. J. Hoffman, "An investigation of filter choice for filtered back-projection reconstruction in PET," IEEE Transactions on Nuclear Science, vol. 45, pp. 1133 - 1137, 1998.
[4] H. M. Hudson and R. S. Larkin, "Accelerated image reconstruction using ordered subsets of projection data," IEEE Transactions on Medical Imaging, vol. 13, pp. 601 - 609, 1994.

[5] A. Raheja, T. F. Doniere, and A. P. Dhawan, "Multiresolution expectation maximization reconstruction algorithm for positron emission tomography using wavelet processing," IEEE Transactions on Nuclear Science, vol. 46, pp. 594 - 602, 1999.

[6] P. Kisilev, M. Jacobson, and Y. Y. Zeevi, "Wavelet domain ML reconstruction in positive emission tomography," Proceedings of 22nd Annual International Conference of the IEEE Engineering in Medicine and Biology Society, pp. 90-93, 2000

[7] B. A. Mair, R. B. Carroll, and J. M. M. Anderson, "Filter banks and the EM algorithm," Proceedings of Nuclear Science Symposium, pp. 1747 - 1751, 1996.

[8] P. Andrey and P. Tarroux, "Unsupervised segmentation of Markov random field modeled textured images using selectionist relaxation," IEEE Transactions on Pattern Analysis and Machine Intelligence, vol. 20, pp. 252 - 262, 1998.

[9] C. Germain, J. P. D. Costa, and P. Baylou, "Multiscale estimation of textural features. Application to the characterization of texture anisotropy," Proceedings of 15th International Conference on Pattern Recognition, pp. 923-926, 2000.

[10] F. Meyer and R. R. Coifman, "Brushlets: A tool for directional image analysis and image compression,” Applied and computational harmonic analysis, vol. 4, pp. 147-187, 1997.

[11] D. Donoho, "Nonlinear solution of linear inverse problems by wavelet-vaguelette decompositions," Journal of Applied and Computational Harmonic Analysis, vol. 2, pp. 101-126, 1995.

[12] S. Guedon and J. Kalifa, "Medical application of denoising in wavelet basis," Department of Biomedical Engineering, Columbia University, New York, Research Report 2001.

[13] D. L. Donoho and I. M. Johnstone, "Ideal spatial adaptation via wavelet shrinkage,” Biometrika, vol. 81, pp. 425-455, 1994.

[14] S. Mallat, A Wavelet Tour of Signal Processing. San Diego, CA: Academic Press, 1998.

[15] E. D. Angelini and A. Laine, "Spatio-temporal directional analysis of real-time three dimensional cardiac ultrasound," in Wavelets in Signal and Image Analysis, F. Meyer and A. Petrosian, Eds.: Kluwer Academic, 2001.

[16] S. G. Chang, B. Yu, and M. Veterlli, "Spatially adaptive wavelet thresholding with context modeling for image denoising," Proceedings of International Conference on Image Processing, Chicago, IL, 1998.

[17] M. T. Chan, R. M. Leahy, E. U. Mumcouglu, S. R. Cherry, J. Czernin, and A. Chatziioannou, "Comparing lesion detection performance for PET image reconstruction algorithms: a case study,” IEEE Transactions on Nuclear Science, vol. 44, pp. 1558 - 1563, 1997. 